\title{
A Just Noticeable Difference-Based Video Quality Assessment Method with Low Computational Complexity
}

\author{
Woei-Tan Loh ${ }^{1} \cdot$ David Boon Liang Bong ${ }^{1}$
}

Received: 10 November 2017 / Revised: 6 July 2018 / Published online: 6 August 2018

(c) Springer Science+Business Media, LLC, part of Springer Nature 2018

\begin{abstract}
A Just Noticeable Difference (JND)-based video quality assessment (VQA) method is proposed. This method, termed as JVQ, applies JND concept to structural similarity (SSIM) index to measure the spatial quality. JVQ incorporates three features, i.e. luminance adaptation, contrast masking, and texture masking. In JVQ, the concept of JND is refined and more features are considered. For the spatial part, minor distortions in the distorted frames are ignored and considered imperceptible. For the temporal part, SSIM index is simplified and used to measure the temporal video quality. Then, a similar JND concept which comprises of temporal masking is also applied in the temporal quality evaluation. Pixels with large variation over time are considered as not distorted because the distortions in these pixels are hardly perceivable. The final JVQ index is the arithmetic mean of both spatial and temporal quality indices. JVQ is found to achieve good correlation with subjective scores. In addition, this method has low computational cost as compared to existing state-of-the-art metrics.
\end{abstract}

Keywords Video quality $\cdot$ Just noticeable difference $\cdot$ Temporal $\cdot$ Computational complexity

\section{Introduction}

Just noticeable difference (JND) is defined as the amount of variation of stimuli for the human visual system (HVS) to perceive. The concept originates from the field of psychophysics. This limen, known as the onset of the psychological reactions, is well-known for signal processing, especially image processing. In general, the JND concept is categorized into subband and pixel based [1]. Subband JND involves the frequency domain and usually uses either the discrete cosine transform (DCT) or the wavelet transform. It takes consideration of contrast

David Boon Liang Bong

davidblbong@yahoo.com; bbldavid@unimas.my

1 Faculty of Engineering, Universiti Malaysia Sarawak, Kota Samarahan, Malaysia 